\section{Blocking SIRPa could restore macrophage appetite and mitigate pneumonia}

humans and
mice show
reduced
phagocytic
capacity
following the
resolution
of acute
inflammation

Patients who have been hospitalized with sepsis or trauma have a much higher risk of developing hospital-acquired pneumonia and this is often fatal. Reporting in Nature Immunology, Roquilly et al. show that mononuclear phagocytes from both humans and mice show reduced phagocytic capacity following the resolution of acute inflammation. The authors link this paralysis of macrophages to the inhibitory receptor SIRPa and suggest that targeting this pathway could help protect patients from hospital-acquired pneumonia.

Patients who are recovering from a critical medical condition have a $30-50 \%$ risk of developing hospital-acquired pneumonia, and there is growing consensus that this may be due to immunosuppression that develops following systemic inflammation. In initial experiments, Roquilly et al. examined the phagocytic capacity of monocytes taken from patients who were recovering from trauma-induced systemic inflammation. Compared

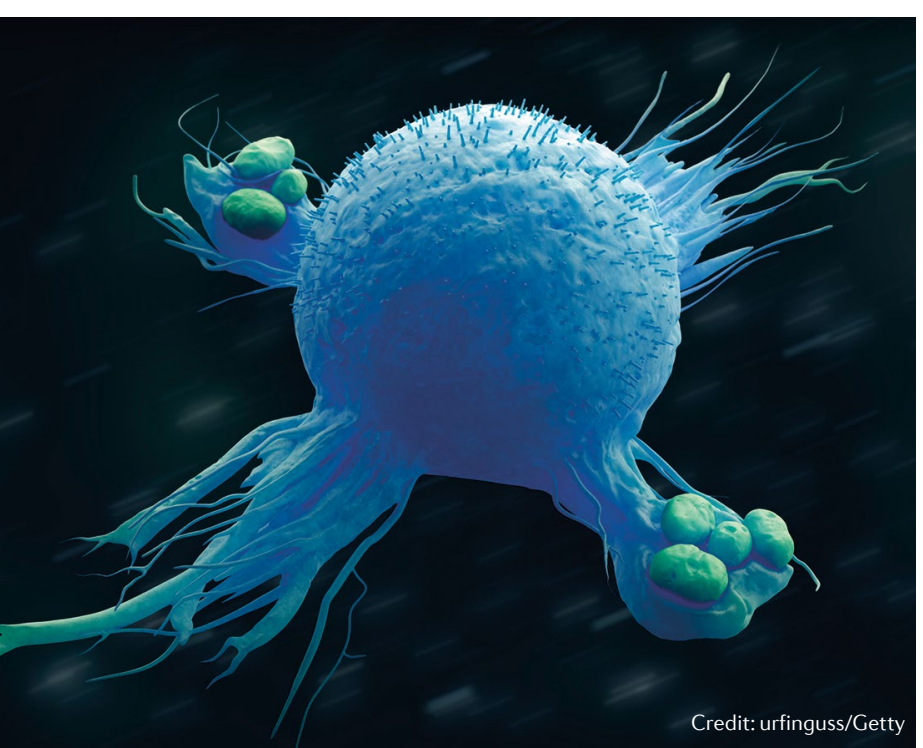

with monocytes from healthy controls, a lower proportion of monocytes from these patients were able to phagocytose Escherichia coli and Staphylococcus aureus bacteria, even 6 months after the resolution of inflammation. Monocytes from patients who had recovered from sepsis showed a similar reduction in phagocytic capacity.

The authors explored this phenomenon further in mouse models, where mice that had received a primary lung infection with $E$. coli, S. aureus or influenza A virus (IAV) were allowed to recover for 7 days and then were infected with $E$. coli or $S$. aureus to cause a secondary pneumonia. Mice that received a secondary bacterial infection had higher bacterial burdens than mice receiving a primary challenge with the same pathogen. Similarly to what had been observed in human monocytes, this was associated with impaired phagocytic capacity of alveolar macrophages during secondary infections. Tracing experiments suggested that the 'paralysed' alveolar macrophages seen in secondary infections were not derived from circulating precursors but were either the lung-resident macrophages that had been present during the primary challenge or the progeny of these cells.

The transfer of paralysed alveolar macrophages into naive recipient mice restored their phagocytic capacity, suggesting that endogenous mediators in the tissue microenvironment induce the paralysis programme and not the infection itself. Notably, deletion of regulatory $\mathrm{T}$ cells or impairment of TGF $\beta$ receptor signalling did not restore phagocytic capacity in paralysed macrophages. In addition, exposure to HMGB1 (a prototype damage-associated molecular pattern) did not reduce the phagocytic capacity of alveolar macrophages in mice; however, exposure to TNF had a minor suppressive effect. The authors observed similar epigenetic remodelling in paralysed alveolar macrophages to what has previously been reported in macrophages 'trained' by exposure to lipopolysaccharides or $\beta$-glucan, and they detected a major role for NF- $\kappa \mathrm{B}$ in this epigenetic reprogramming.

Closer scrutiny of the molecular basis of alveolar macrophage paralysis showed that SIRPa, which is a known inhibitor of phagocytosis, was upregulated in the alveolar macrophages of mice that had resolved a primary bacterial or viral infection. SIRPa is activated by the surfactant proteins SP-A and SP-D, and the authors detected increased concentrations of SP-D (but not SP-A) in the bronchoalveolar lavage of infected mice. The phagocytic activity of alveolar macrophages during secondary pneumonia was markedly improved in SIRPa-deficient mice. Interestingly, SIRPa seemed to have a direct inhibitory effect on alveolar macrophages early in the course of infection, but other mediators produced secondary to SIRPa activation maintained macrophage paralysis by creating a suppressive microenvironment.

Returning to patients with systemic inflammation, the authors showed that blocking antibodies against SIRPa restored the capacity of their monocytes to phagocytose E. coli ex vivo. These findings may be even more relevant clinically in the current climate where many patients are being hospitalized with pneumonia as a result of the SARS-CoV-2 pandemic.

Yvonne Bordon

ORIGINAL ARTICLE Roquilly, A. et al. SIRPa triggers tolerogenic training of resident alveolar macrophages after inflammation, leading to immune paralysis and increased susceptibility to secondary pneumonia. Nat. Immunol. https:// doi.org/10.1038/s41590-020-0673-x (2020) 